\title{
Evaluación del desempeño docente para la educación superior en tiempos de Covid-19
}

\author{
Evaluation of teaching performance for higher education in times of Covid-19
}

Avaliação do desempenho docente para o ensino superior em tempos de Covid-19

\section{ARTÍCULO GENERAL}

\begin{tabular}{|c|c|c|c|}
\hline $\begin{array}{c}\text { Juana Alvarado Estrada } \\
\text { https://orcid.org/0000-0002- } \\
2622-3873\end{array}$ & $\begin{array}{c}\text { Sonia Cassano Tasaico } \\
\text { https://orcid.org/0000-0002- }\end{array}$ & $\begin{array}{c}\text { Heleny Terán Plasencia } \\
\text { https://orcid.org/0000-0003- }\end{array}$ & $\begin{array}{c}\text { Héctor Navarro Monroy } \\
\text { https://orcid.org/0000-0001- }\end{array}$ \\
\hline$\underline{2622-3873}$ & $\underline{3421-3616}$ & 4637-1061 & 8194-9274 \\
\hline Universidad César Vallejo & Universidad César Vallejo & Universidad César Vallejo & Universidad César Vallejo \\
\hline Lima - Perú & Lima - Perú & Lima - Perú & Lima - Perú \\
\hline
\end{tabular}

Recibido 07 de Abril 2021 | Arbitrado y aceptado 19 de Junio 2021 | Publicado en 03 Setiembre 2021

\begin{abstract}
RESUMEN
La intención del presente artículo es recuperar, analizar y trascender el conocimiento de los distintos instrumentos para evaluar el desempeño docente en tiempos de Covid-19. Teniendo en cuenta la problemática acontecida que ha obligado migrar de una educación presencial a una educación virtual donde las técnicas de evaluación del desempeño debieron adaptarse a la nueva realidad digital. Se procedió a elegir diligentemente las fuentes bibliográficas, seleccionando artículos científicos, tesis y libros enmarcadas en las ciencias sociales.

Por tanto, a lo largo del documento se presentan técnicas utilizadas en la medición del desempeño docente con el objetivo de asegurar la calidad educativa en este escenario de crisis sanitaria por Covid-19. Se abordará desde su conceptualización y fundamentos teóricos de la evaluación del desempeño, competencias del docente virtual, técnicas e instrumentos de medición que sustentan nuestra variable.

Palabras clave: Evaluación del docente, calidad de la educación, educación a distancia.
\end{abstract}

\section{ABSTRACT}

The intention of this article is to recover, analyze and transcend the knowledge of the different instruments to evaluate teaching performance in times of Covid-19. Taking into account the problems that have occurred that have forced migration from face-to-face education to virtual education where performance evaluation techniques had to adapt to the new digital reality. The bibliographic sources were diligently chosen, selecting scientific articles, theses and books framed in the social sciences.

Therefore, throughout the document, techniques used to measure teacher performance are presented in order to ensure educational quality in this scenario of the health crisis caused by Covid-19. It will be approached from its conceptualization and theoretical foundations of performance evaluation, virtual teacher competencies, techniques and measurement instruments that support our variable.

Keywords: Teacher evaluation, quality of education, distance education.

\section{RESUMO}

A intenção deste artigo é resgatar, analisar e transcender o conhecimento dos diferentes instrumentos de avaliação do desempenho docente em tempos de Covid-19. Tendo em conta os problemas ocorridos que forçaram a migração do ensino presencial para o ensino virtual onde as técnicas de avaliação de desempenho tiveram de se adaptar à nova realidade digital. As fontes bibliográficas foram escolhidas com diligência, selecionando artigos científicos, teses e livros enquadrados nas ciências sociais.

Por isso, ao longo do documento, são apresentadas técnicas utilizadas para mensurar o desempenho docente a fim de garantir a qualidade educacional nesse cenário de crise sanitária causada pela Covid-19. Será abordado a partir de sua conceituação e fundamentos teóricos de avaliação de desempenho, competências do professor virtual, técnicas e instrumentos de medição que suportam nossa variável.

Palavras-chave: Avaliação docente, qualidade da educação, educação a distância. 


\section{Introducción}

A escala internacional, el brote de Covid-19 iniciado en Wuhan ciudad ubicada en el centro de China a fines del año 2019 y su propagación en todo el mundo, ha ocasionado impactos negativos en distintos sectores, entre los más afectados salud, educación y economía.

En este panorama las instituciones académicas se vieron forzadas a cancelar por completo la enseñanza presencial incluyendo reuniones académicas, seminarios, uso de laboratorios y otras experiencias formativas para mitigar el riesgo que representa el contagio. Según UNESCO (2020) nunca se había sufrido una alteración de tal escala en el campo educativo, con resultados de más del $60 \%$, en 129 países del mundo, quienes tuvieron que adaptar su práctica pedagógica. Por consiguiente, los gobiernos de cada país emanaron normas para la implementación de una educación virtual haciendo frente a los desafíos que esto conlleva hacia una rápida adaptación curricular acorde a la enseñanza remota durante el aislamiento social obligatorio.

En tal sentido Crawford (2020) afirma que se ha incrementado la utilización de herramientas en línea, para facilitar la enseñanza a distancia; los docentes han tenido que adaptarse al cambio abrupto en la aplicación de nuevas estrategias de educación a distancia, capacitándose en la digitalización de sus actividades y realizando esfuerzos para concretizar en proceso de enseñanza aprendizaje, mediante el desarrollo de competencias en el uso de plataformas digitales como: Blackboard, Classroom, Google Meet, , Microsoft Teams, Zoom con la finalidad de poder brindar continuidad a sus labores académicas. Según Giannini (2020) el uso acelerado de distintas plataformas virtuales es el experimento más intrépido en tecnología educativa, no obstante imprevisto y no planificado. La evaluación de los resultados es una necesidad imperante para reafirmar la inclusión, innovación y cooperación en la enseñanza superior.

A juzgar por Toffler (1979, como se citó en Cano 2016) destaca la siguiente premisa "Los analfabetos del siglo XXI no serán aquellos que no sepan leer y escribir, sino aquellos que no sepan aprender, desaprender y reaprender a partir de la información digital" (p. 117). Existe una alta probabilidad que, en los años venideros, el escenario de la educación superior sufra cambios relevantes en donde los entornos virtuales serán una herramienta complementaria imprescindible. Puesto que la enseñanza virtual ofrece un ambiente cómodo y dinámico en donde la transferencia de información es inmediata y eco sustentable. Sin embargo, presenta algunas desventajas como escasa interrelación 
entre pares al ser una actividad muy solitaria. En consecuencia, es necesario que los educadores cuenten con una serie de competencias que los guíen a atender las demandas actuales que exige el nuevo contexto social. Escudero et al. (2017) aseveran que, los docentes son entes claves en el mejoramiento de la calidad educativa (p.34). Por tanto, su rol es esencial y se manifiesta en el optimo desempeño de su actividad pedagógica.

La nueva normalidad demanda docentes idóneos, que desarrollen según Salinas (2017) ciertas competencias básicas en un entorno de educación virtual: pedagógicas, sociales y digitales (p. 58). Las habilidades y destrezas pedagógicas acreditan al docente en su desenvolvimiento con eficiencia, tomando decisiones con idoneidad ante circunstancias imprevistas. Las capacidades sociales buscan relaciones sanas, nutriendo y engrandeciendo el espacio social fundamentales en la práctica docente. Las competencias digitales están relacionadas a las habilidades tecnológicas necesarias para gestionar el aprendizaje a distancia. Según Álvarez (2020) se podrá lograr la calidad de la práctica de la enseñanza si se mejoran, implementan los planes de estudio y si se adecúan también los textos educativos; sin embargo, está claro que, sin docentes competentes y comprometidos, el perfeccionamiento real de la educación no tendrá lugar nunca (p. 25). En consecuencia, nace la exigencia de reflexionar sobre la evaluación del desempeño docente en entorno virtual y valorar su impacto con la finalidad de implantar estrategias de mejora ante el reto de una educación no presencial.

A lo largo del documento se presentan técnicas utilizadas en la medición del desempeño docente con el objetivo del aseguramiento una educación de calidad en el contexto de crisis sanitaria por Covid-19. Se abordará desde su conceptualización y fundamentos teóricos de la evaluación del desempeño, competencias del docente virtual, técnicas e instrumentos de medición que sustentan nuestra variable.

\section{Método}

El presente artículo se cimienta en una revisión documental amplia que comprende la exploración de diversos artículos científicos arbitrados, tesis y libros digitales de Ciencias Sociales de Scielo, Dialnet y Redalyc indexadas en bases de datos electrónicas como Scopus, Sciencedirect, Springer, sobre la evaluación del desempeño docente, dentro de un margen temporal de cinco años desde 2016 hasta el 2020.

Los descriptores utilizados en la presente investigación son: Evaluación del docente, calidad de la educación y educación a distancia, las cuales permitieron dar inicio al proceso heurístico en la identificación y revisión exhaustiva de 58 fuentes 
documentales en español, inglés, portugués e italiano, seleccionando 41 de acuerdo con su pertinencia discursiva en relación con la variable de estudio, mediante los siguientes criterios excluyentes: Aplicando la técnica hermenéutica de investigación se logró interpretar y analizar los documentos recabados para el desarrollo de los núcleos temáticos y la construcción teórica que forman parte del estado del arte en la generación de nuevos conocimientos y comprensión crítica del fenómeno en análisis.

\section{Desarrollo}

\section{Conceptualización del Desempeño Docente}

La calidad de la educación configura diversos factores importantes, uno de ellos es el desempeño docente, que resulta trascendental para enfrentar los nuevos retos que implican diferentes desafíos sociales, culturales y tecnológicos ocurridos a raíz del aislamiento obligatorio ocasionado por Covid-19. Frente a la problemática, las demandas y los desafíos han dejado entrever que existen debilidades en la praxis del docente. El desempeño docente, según Martínez Rizo (2016) "es un elemento imprescindible asociado de forma directa a la calidad educativa" (p. 3), por tanto, los gobiernos: nacional, regional y local tienen como propósito aplicar estrategias apropiadas y pertinentes en un contexto virtual y que con el apoyo de la Organización de las Naciones Unidas para la Educación, la Ciencia y la Cultura y otros organismos privados se viene brindando capacitación a los docentes sobre herramientas digitales (UNESCO, 2020).

La evolución histórica de la definición de la evaluación del desempeño docente se manifiesta desde Tyler (1950) quien la define como un proceso que consiste en la comprobación del logro de conocimientos (p. 34). A lo largo de las décadas, el concepto se extiende y contempla a las metas y objetivos institucionales tal como Harris (1986) afirma, proceso de verificación de las acciones del evaluado en la contribución satisfactoria al logro de los objetivos totales (p. 276). La ética profesional es relevante en la evolución del concepto tal como evidencia Gil et al. (1997) apreciar con la mayor objetividad posible, la actuación de una persona durante un determinado tiempo, en relación con su trabajo actual y sus características personales, así como su contribución a objetivos previstos (pp.129 - 130). Surge la necesidad que la evaluación se realice con frecuencia para el aseguramiento de la calidad en la educación, según Chiavenato (1999) es la observación sistemática del desempeño de cada persona en el cargo o del potencial de desarrollo futuro con el fin de estimular o juzgar el valor, la excelencia, las cualidades 
de alguna persona. (p. 357). Con la finalidad de contar con formatos de evaluación estandarizada Sartre y Aguilar (2003) considera que debe ser un proceso sistemático y estructurado de seguimiento de la labor profesional para valorar su actuación y los resultados logrados en el desempeño de su cargo (p. 321). La evaluación del desempeño conforme a Castillo y Cabrerizo (2009) es un proceso de acompañamiento, regulación, guía y sistematización de los aprendizajes, para mejorar el proceso de enseñanza aprendizaje (p. 22). Por tanto, la medición del desempeño docente debe considerar diversas habilidades, competencias y valores, en esta línea Martínez Ruiz y Lavín (2017) mencionan que es una actividad inherente a la profesión docente y considera ciertos elementos como parte de su práctica de enseñanza (p. 3). En este sentido, las herramientas tecnológicas contribuyen en el fortalecimiento de la evaluación del desempeño docente, tal como sostiene García (2020) es el seguimiento de la formación continua del docente y la aplicación de la tecnología como elemento clave para sustentar la calidad (p. 9).

En definitiva, el concepto del desempeño docente ha evolucionado con el pasar de los años, en sus inicios se centraba en el logro de objetivos y en los últimos años, el uso de la tecnología se ha convertido en un elemento relevante a tomar en consideración en palabras de Martínez Rizo (2016) asevera que "el fin último de la medición del desempeño de la actuación del docente es la mejora de la calidad de la docencia y favorecer el aprendizaje de los estudiantes" (p. 15). Por otro lado Phillips (2018) afirma que "la evaluación educativa es fundamental porque permite comprender la situación de los estudiantes, docentes y sobre todo del servicio que se ofrece, así mismo, identificar los resultados para la toma de decisiones en mejora del sistema educativo" (p.15), teniendo como meta la retroalimentación para el docente, promoviendo capacitaciones y actualizaciones permanentes, sirviendo como un estímulo para mejorar su trayectoria formativa y por ende la calidad de la educación, debiendo entenderse que la evaluación del desempeño docente significa evaluar el nivel de cumplimiento de las funciones y la calidad de éstas concretizadas en los logros obtenidos. 
Tabla 1

Evolución de la Evaluación del Desempeño

\begin{tabular}{|c|c|c|}
\hline Autor & Año & Concepto \\
\hline Tyler & 1950 & $\begin{array}{l}\text { Proceso que consiste en la comprobación del logro de conocimientos } \\
\text { (p. 34). }\end{array}$ \\
\hline Harris & 1986 & $\begin{array}{l}\text { Proceso de verificación si las acciones del evaluado contribuyen } \\
\text { satisfactoriamente al logro de los objetivos totales (p. 276). }\end{array}$ \\
\hline $\begin{array}{l}\text { Gil, Ruiz y } \\
\text { Ruiz }\end{array}$ & 1997 & $\begin{array}{l}\text { Apreciar con la mayor objetividad posible, la actuación de una } \\
\text { persona durante un determinado tiempo, en relación con su trabajo } \\
\text { actual y sus características personales, así como su contribución a } \\
\text { objetivos previstos (pp.129-130). }\end{array}$ \\
\hline Chiavenato & 1999 & $\begin{array}{l}\text { Observación sistemática del desempeño de cada persona en el cargo o } \\
\text { del potencial de desarrollo futuro con el fin de estimular o juzgar el } \\
\text { valor, la excelencia, las cualidades de alguna persona. (p. 357). }\end{array}$ \\
\hline $\begin{array}{l}\text { Sartre y } \\
\text { Aguilar }\end{array}$ & 2003 & $\begin{array}{l}\text { Proceso sistemático y estructurado de seguimiento de la labor } \\
\text { profesional para valorar su actuación y los resultados logrados en el } \\
\text { desempeño de su cargo (p. 321). }\end{array}$ \\
\hline $\begin{array}{l}\text { Castillo y } \\
\text { Cabrerizo }\end{array}$ & 2009 & $\begin{array}{l}\text { Proceso de acompañamiento, regulación, guía y sistematización de los } \\
\text { aprendizajes, para mejorar el proceso de enseñanza/aprendizaje (p. } \\
\text { 22). }\end{array}$ \\
\hline $\begin{array}{l}\text { Martínez } \\
\text { Ruiz y } \\
\text { Lavín }\end{array}$ & 2017 & $\begin{array}{l}\text { Actividad inherente a la profesión docente y considera ciertos } \\
\text { elementos como parte de su práctica de enseñanza (p. } 3 \text { ). }\end{array}$ \\
\hline García & 2020 & $\begin{array}{l}\text { Seguimiento de la formación continua del docente y la aplicación de la } \\
\text { tecnología como elemento clave para sustentar la calidad (p. 9). }\end{array}$ \\
\hline
\end{tabular}

Fuente: Elaboración propia

\section{Calidad de la educación}

El académico Otto Granados Roldán, presentó el 12 de octubre 2018 en la Universidad Autónoma de Aguascalientes su obra "Reforma Educativa", publicación creada para divulgar un diseño, formulación e instrumentación que asegure la mejora de una educación de calidad, llevando a la reflexión sobre las principales circunstancias, desafíos, metas que requieren los nuevos tiempos con el desarrollo de nuevas tecnologías, la inteligencia artificial y robótica. La calidad en educación se concibe como un criterio de la integración, interrelación y desarrollo de diversos componentes que aseguran el cumplimiento de objetivos de aprendizaje y se manifiestan con la formación de sujetos críticos, innovadores, reflexivos y democráticos preparados para afrontar la vida profesional.

Granados (2018) define a la calidad de la educación como "un constructo que contiene un conjunto de condiciones de diversa naturaleza que garantizan, en su interrelación, el buen funcionamiento de una institución educativa" (p. 103). El contexto 
actual de constantes transformaciones a nivel mundial demanda condiciones de productividad en un marco de globalización que implica la calidad de las entidades educativas de nivel superior para una sólida formación de los educandos en beneficio de la sociedad.

\section{Evaluación del desempeño}

En la historia de la humanidad, desde 221 a.C. se habrían ejecutado las primeras evaluaciones del desempeño para la escuela en China y con el transcurrir del tiempo este tema ha ganado importancia, además de haberse convertido muy complejo dada la necesidad de buscar la calidad en el sector educativo, surgiendo en el tiempo diversas concepciones de evaluación del desempeño y autores preocupados por regularizar y orientar la conducta de los sujetos dentro de las instituciones para fortalecer y elevar la calidad en el proceso educativo que respondan a las nuevas exigencias del mundo, como exhortaba Freire (2017), "la dimensión política no puede estar desconectada de la dimensión personal" (p. 67), es decir, el contexto y mediatez global debe guiar la formación docente, quienes tienen que estar conscientes de su inconclusión para ir en busca de la superación de sus limitaciones y nuevos conocimientos y además, la evaluación no puede terminar con la libertad y autonomía del educador.

Uno de los discípulos de Paulo Freire, pedagogo, director y fundador de Institutos, Romao (2019) manifiesta que la evaluación se basa en un elemento político de exclusión; cuantificando los desempeños con la finalidad de clasificar, excluyendo lo que no parece, lo frágil o lo que no convence, y un elemento político de inclusión; que, a diferencia del primero, también ayuda a identificar lo frágil, pero con el propósito de diagnosticar para prever, tomar decisiones y solucionar las carencias encontradas en el desempeño (pp. 3439). Los docentes se sienten amenazados y muchas veces en estos procesos selectivos y discriminatorios se ha encontrado resistencia ante las evaluaciones creando controversias de su legitimidad científica. En esta modalidad la evaluación identifica lo bueno y malo para premiarlos o castigarlos confirmando su objetivo excluyente. La evaluación se aplica a tres campos de acción: evaluación del aprendizaje; alude a la comprobación de los resultados de los estudiantes presumiendo que ellos son los únicos que aprenden en la escuela superior, cuando se sabe que los docentes en su praxis de enseñar también aprenden. Evaluación del desempeño; dirigida a los docentes, encargados de la educación, quienes aun sintiéndose discriminados y pese a su resistencia, están sujetos a evaluaciones establecidas y monitoreos permanentes para ser o extender su acreditación como 
profesional de la educación. Y, como nos regimos por la meritocracia, ellos están obligados a registrar sus nuevos estudios o capacitaciones siendo actualizados periódicamente, además de, alienarse a la producción académica o investigación científica atendiendo posiblemente a los rankings de las instituciones superiores y no tanto a la resolución de la problemática que afecta a la humanidad en revistas científicas en la Base Qualis. El otro campo de acción de la evaluación es la institucional; suministrada en la educación superior, asignada para lograr capacidades formativas y productoras de conocimientos y no sólo como proceso de comprobación de la praxis de los docentes, además, del éxito de los egresados en los diferentes campos laborales al servicio de la población (Romao, 2019, pp. 40-48).

\section{Competencias a evaluar en el desempeño}

Romao (2018) plantea que la interacción, solidaridad, colaboración y dialogicidad son elementos fundamentales para la ejecución de la evaluación del desempeño donde los saberes se relacionan con las competencias pedagógicas, sociales y digitales, importantes para la ejecución de la evaluación, legitimando los conocimientos en estos tiempos que la digitalización es de gran albergadura, reconociendo que la evaluación tiene función de diagnóstico, pero si buscamos solamente conocer ¿cómo se desempeñó el docente?, o sea, con el fin de emitir un juicio, entonces tendrá función clasificatoria (p. 41).

\section{Competencias Pedagógicas}

El educador debe adaptarse a los retos de la sociedad contemporánea en palabras de García (2020) "la tarea del docente no se limita al desarrollo del área cognitiva de los educandos, además, demanda ser mediador del aprendizaje, organizador del trabajo en equipo, promotor de la integración e investigador de los fenómenos educativos de sus contextos" (p.34). Facilitan al educador desenvolverse de forma eficiente, respondiendo con inmediatez frente a escenarios imprevistos. A continuación, las competencias pedagógicas a evaluar para la medición del desempeño docente:

-Planificación pedagógica: Comprende los objetivos, como guía del proceso formativo de enseñanza. Realizar un diagnóstico a los estudiantes, con la finalidad de conocer la situación real, en función de los conocimientos, necesidades y dificultades del alumnado. Brindar asesoría a los alumnos sobre hábitos de estudio sugeridos para el aprendizaje a distancia. 
-Gestión del conocimiento: Consiste en seleccionar contenidos pertinentes para el curso. Proporcionar una retroalimentación precisa y oportuna a cerca de las actividades que realice el estudiante; planificar, diseñar y aplicar evaluaciones tanto diagnósticas, aplicativas o sumativas.

-Competencia curricular: Capacidad de aplicar y enseñar la materia a cargo. Hace referencia al dominio, experiencia y conocimientos actualizados en el uso de método, herramientas y guías formativas estandarizadas propias de la disciplina que dirige.

\section{Competencias Sociales}

Dada las circunstancias de aislamiento por Covid-19, es probable que los alumnos lleguen a desarrollar sentimiento de soledad y abandono. A juzgar por Palacios et al. (2019) las competencias sociales cimentan el desempeño docente acorde al contexto (p.24). Por tanto, el docente debe poseer habilidades necesarias para orientar el proceso de enseñanza aprendizaje a un entorno amigable.

Buscar relaciones sanas, nutriendo y engrandeciendo el espacio social es fundamental en la práctica docente. Goleman (2018) las habilidades sociales permiten establecer contacto con los demás de forma profunda (p.53). El docente debe llegar tocar el corazón de sus estudiantes para que estos se sientan cómodos dentro de un espacio educativo virtual que puede percibirse impersonal y frio.

Las competencias sociales que debe presentar el profesor según Achcaray y Gámez (2018) son empatía social, comunicación asertiva y motivación hacia el logro (p.34).

- Empatía social: Procurar la debida atención a cada uno de los estudiantes. Valorar las condiciones individuales tratando de ponerse en su lugar y teniendo en cuenta sus opiniones.

- Comunicación asertiva: El docente debe expresarse con claridad, seguridad, respetando al estudiante, Utilizar una comunicación abierta, positiva y constructiva. Promover un ambiente de respeto en el vínculo docente discente procurando evitar la ironía. Fomentar en los estudiantes a expresar en libertad su sentir en relación con los diferentes tópicos de la asignatura.

- Motivación hacia el logro: Estimular y favorecer el trabajo cooperativo en un clima virtual de enseñanza aprendizaje idóneo. Generar y preservar equipos de aprendizaje social y amigable en donde los estudiantes se encuentren cómodos. Incentivar a los educandos permanentemente para que su rendimiento académico no 
disminuya durante el proceso de aprendizaje puesto que ante la situación actual no se deteriore al no sentirse guiados por el maestro.

\section{Competencias Digitales}

La situación sanitaria y social a nivel mundial ha puesto en evidencia según Balzola (2020) "la falta de formación y experiencia de los profesores en las habilidades tecnológicas necesarias para gestionar el aprendizaje a distancia" (p.25). La virtualización de la enseñanza de forma apresurada requiere de capacidades y destrezas digitales en la resolución de problemas referente a la praxis educativa. Scull et al. (2020) cambiar de manera imprevista de una educación presencial a una completamente en línea no ha sido fácil, sin embargo, el contar con diversas herramientas tecnológicas para optimizar la participación de los estudiantes puede contribuir en la adaptación al nuevo horizonte educativo (p. 7).

Las competencias digitales son a juzgar por Durán et al. (2016) "habilidades, conocimientos y actitudes para utilizar adecuadamente la tecnología incluyendo tanto los ordenadores como los diferentes programas e internet que permiten la búsqueda, acceso, organización y utilización de la información con el fin de construir conocimiento" (p. 98). Los docentes tienen la responsabilidad de capacitarse en el dominio de las tecnologías de información y comunicación (TICs) puesto que cambian y se desarrollan con celeridad. Según el Instituto Nacional de Tecnologías Educativas y de Formación del profesorado (2017) establece cinco aspectos que integran la competencia digital educador (p. 3):

- Búsqueda de Información: Se trata de la navegación, selección, evaluación, almacenamiento de y recuperación de datos y contenidos virtuales.

- Difusión de datos: Compartir y socializar los contenidos digitales mediante la participación en línea. Considerando la Netiqueta, Gestión de la identidad digital.

- Desarrollo de contenidos digitales: La creación, integración y reelaboración de datos digitales, así como los derechos de autor y licencias.

- Seguridad digital: Protección de identidad digital, datos personales, de la salud mental y ambiente.

- Resolución de problemas: respuestas tecnológicas a las necesidades actuales, creatividad y uso de la tecnología digital para la innovadora. 


\section{Técnicas e Instrumentos en la Evaluación del Desempeño}

Es necesario tener en cuenta el dominio de los aspectos cualitativos en una evaluación del desempeño, si deseamos corregir, orientar, reformular procedimientos pedagógico-didáctico, como asevera Romao (2019), teniendo el diálogo como factor primordial (p. 38). Existe una progresiva y alta expectativa en la aplicación de las técnicas e instrumentos para la evaluación del desempeño debido a la demanda, especialmente en la educación superior, ajustando las técnicas de observación, entrevista y encuesta virtual como las más asequibles para su propósito.

\section{Figura 1}

Instrumentos en la técnica de observación

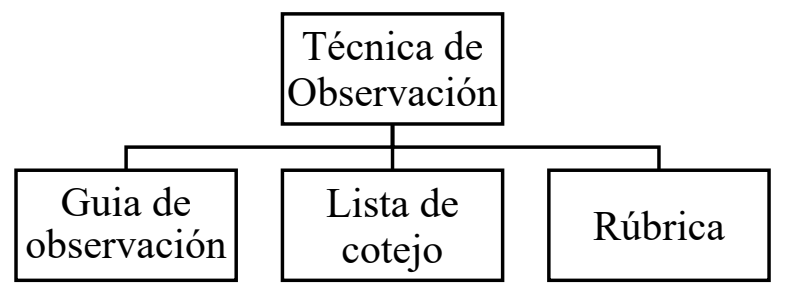

Fuente: Elaboración propia

\section{Técnica de Observación}

La observación se concibe como una percepción, lógica, programada y ordenada de los aspectos vinculados a los propósitos del estudio, el cual, se enmarca en las características frecuentes, con la finalidad de brindar el sustento científico en relación a la esencia de la observación previamente analizada. Se define por Gonzales (2020) a la consolidación de fuentes de conocimiento en forma estructural, organizada, vigente y veraz con una intención científica (p. 14).

La técnica de observación para Leiva et al. (2016) es una didáctica pedagógica, de mejora continua que favorece la retroalimentación, en la evaluación de la práctica pedagógica (p. 3). Es un método de evaluación con un propósito definido a la fuente de información, mediante la percepción adecuada del escenario y las condiciones determinadas. Según Cohen y Goldhaber (2016) las mediciones observacionales vienen a ser un elemento preciso y necesario de un procedimiento de evaluación, puesto que las situaciones son cambiantes y particulares (p. 7).

En ocasiones, la técnica de observación puede tender a la subjetividad, por tanto, 
se deben registrar criterios puntuales y bien definidos para su tratamiento. A juzgar por Kidd y Murray (2020) señala que la técnica de observación se refiere a contrastar la realidad actual, con la finalidad de entenderla en todo sentido y generar resultados concretos (p. 28). Se deben evitar los prejuicios, estereotipos, críticas anticipadas o crea una relación de adversarios entre el observador y el docente evaluado, puesto que, no favorece el mejoramiento del desempeño docente.

Los instrumentos empleados en la técnica de observación para la evaluación de las competencias del docente en tiempos de Covid-19 son: la guía de observación, lista de cotejo y rúbrica.

\section{Instrumento: Guía de observación}

La guía de observación para Lezcano y Vilanova (2017) es un conjunto de interrogantes generadas tomando en cuenta la objetividad e las suposiciones elaboradas con precisión para mostrar y guiar la observación (p. 44). El autor propone realizar una relación de indicadores necesarios para enmarcarse como aseveraciones o interrogantes para orientar la guía de observación, indicando las razones que son importantes para el supervisor. Gunn (2018) afirma que, su sistematización contempla aspectos que se pretende anticipar en relación al objeto (p. 33). Es decir, diseñar los objetivos de observación a manera que favorezcan el orden del proceso de supervisión, la permanencia y las veces que se repiten los registros mediante una secuencia con temporalidad, funcional y accesible para un adecuado proceso de análisis para un escenario especifico.

La funcionalidad del instrumento estará sujeta al tipo de monitoreo en el cual se emplee. Al respecto Paz (2019) agrega que las guías de observación permiten operacionalizar la obtención de datos confiables mediante el planteamiento de indicadores que evidencien en el caso del desempeño docente, las competencias de los educadores (p. 8). Es oportuno considerar en el encabezado del documento datos básicos como: la fecha en que se realizó la observación, la hora, identificación del supervisor, nombre del docente a evaluar, enlace web para el acceso a la plataforma virtual, curso, ciclo, sección, entre otros. Es recomendable además dejar un espacio final para anotaciones de hechos importantes y que quizá no encontraban contemplados.

\section{Instrumento: Listas de cotejo}

Los educadores que participan en la modalidad de enseñanza a distancia, debido a 
las restricciones sanitarias impuestas por el estado peruano, deben ser supervisados con la finalidad de medir su desempeño docente en tiempos de Covid-19. Resulta eficaz utilizar una lista de cotejo por parte del evaluador que observa la sesión de clase virtual con la finalidad de dejar evidencia de las competencias desarrolladas para alcanzar una educación de calidad.

La lista de cotejo según Martínez y Guevara (2018), es una herramienta estructurada, que contiene una lista de criterios determinados, los cuales exclusivamente califican la presencia o ausencia de estos a través de una escala dicotómica (p. 26). Durante su aplicación, el supervisor deberá verificar cada uno de los atributos considerados en el instrumento y aplicados o no por el docente en observación.

Burnage (2018) afirma que los resultados obtenidos permiten al evaluador conocer el nivel de desempeño docente y brindar un seguimiento a aquellos considerados "en proceso" con la intención de favorecer en su práctica pedagógica (p. 32). Es indispensable efectuar una retroalimentación a los docentes, posterior a la observación de la clase virtual para que tenga conocimiento de los aciertos y desaciertos detectados además de esclarecer los logros deseados en su desempeño laboral. Para Saucedo, (2018) una lista de cotejo puede utilizarse para evaluar aspectos actitudinales del docente ( $\mathrm{p}$. 18). Es posible considerar aspectos actitudinales en los indicadores para la medición de conducta del evaluado.

Según Geier (2020) los elementos que componen una lista de cotejo son el objetivo, dimensiones, indicadores, escala, instrucciones y como apartado adicional observaciones (p. 33). El objetivo de evaluación se define con un verbo de acción y propósito de la evaluación sobre los criterios a evaluar sobre el desempeño docente. Las Dimensiones son descripciones generales de lo que se desea evaluar. Los indicadores se desprenden a partir de los criterios de evaluación mencionados en las dimensiones, se recomienda que su redacción sea breve, precisa y directa, de modo que permita su observación, sin ambigüedades ni posibles interpretaciones subjetivas. La escala hace referencia a la presencia o ausencia de los indicadores, se registra en la escala dicotómica, en donde se toman únicamente dos valores. Tales como: Si / no; Logrado / No logrado; Cumple / No cumple; Aceptable / Inaceptable entre otros. Las instrucciones, serán un espacio con las indicaciones de dónde y cómo marcar opciones en el instrumento de evaluación. Las observaciones, son apartado opcional para realizar comentarios en general, sugerencias a modo de retroalimentación para el docente 
evaluado.

A juzgar por Lia (2016) entregar anticipadamente, la lista de cotejo a los docentes les permitirá a estos, conocer los criterios esperado durante la evaluación de desempeño (p. 22). Por consiguiente, un docente informado sobre las competencias esperadas en su desempeño podrá priorizar tareas, administrar sus tiempos y asumir con responsabilidad su rol de facilitadores del conocimiento.

\section{Instrumento: la rúbrica}

Con la finalidad de promover cultura de calidad y excelencia es necesario evaluar el desempeño para generar conocimiento y sobre todo crear valor en el docente utilizando diversos instrumentos como la rúbrica; es una escala descriptiva donde se plasma el grado de cumplimiento de los atributos que se pretende valorar. En una tabla se presentan dos ejes, uno vertical; donde se colocan los criterios a evaluar y representa lo que los docentes dominan y otro eje horizontal; donde se estipulan los rangos de valor para evaluar los criterios. Según Dorantes y Tobón (2017), “las rúbricas evidencian niveles de desempeño y desarrollo continuo, posibilitando la identificación de las necesidades, pronostican las dificultades que se pueden encontrar en el desempeño y ayudan a revisar sistemática y permanente la actuación del docente" (p. 4). Así mismo, el docente tiene la oportunidad de analizar su praxis para descubrir las condiciones de calidad. Este instrumento clarifica lo que cada nivel representa en la escala de calificación, además, permite la metacognición para tomar decisiones en mejora continua del aprendizaje.

Hernández et al. (2016) hacen referencia que la socioformación, enfoque que se orienta hacia la transformación de las actividades de formación en diferentes campos, en donde se aborda la evaluación socioformativa cuyo fin es que los evaluados optimicen su desarrollo integral mediante la retroalimentación permanente, adaptando estrategias o técnicas e instrumentos como las rúbricas (p. 2-4). Desde este enfoque las rúbricas han tomado mayor relevancia y son una opción para crear procedimientos de evaluación del desempeño que beneficien el progreso en la acción de las personas. Sin embargo, los detalles que se tiene de las rúbricas son escasos y se hace indispensable conocer todos los elementos de estas para respaldar a los educadores en su proceso de transformación y formación, además de realizar una revisión desde la propuesta metodológica que la socioformación brinda a todos los docentes.

Del mismo modo Hernández (2016) propone la "utilización de instrumentos como la rúbrica socioformativa que permita mejorar y atender las necesidades del contexto con 
una evaluación continua" (p. 6), entendiéndola como una modelo de valoración que integra en una coordenada los indicadores de ejecución de una tarea y en la otra una escala, describiendo qué tipología de ejecución sería acorde de ese grado de la escala.

\section{Figura 2}

Instrumentos en la técnica de entrevista

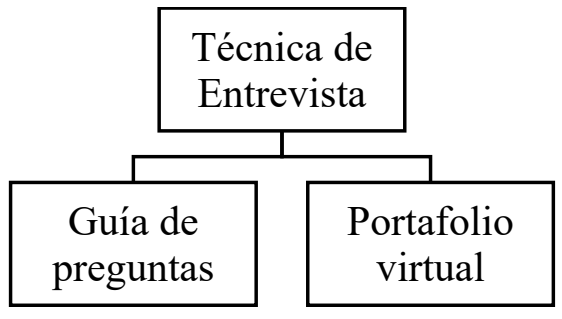

Fuente: Elaboración propia

\section{Técnica: Entrevista}

En estos tiempos, donde se aplica el modelo de aprendizaje por competencias, la evaluación ha llegado a ser un factor muy importante y estratégico en la combinación de conocimientos, habilidades, procedimientos, actitudes y valores para mejorar este sistema de créditos ya que con una prueba objetiva sería insuficiente la evaluación, considerando que el objetivo no es la calificación, sino que el evaluado perciba y esté consciente de lo que aprende. Por ello, el evaluador debe aplicar diversas técnicas, una de ellas es la entrevista; utilizada en diferentes espacios como para la selección de personal, la psicología clínica, la entrevista periodística y en el campo educativo.

Es una técnica parcialmente fácil de utilizar, admite recoger una importante suma de información de los entrevistados y se hace posible acomodar a las características y necesidades de cada uno de ellos. Por lo tanto, es bien aceptada como técnica de evaluación por entrevistados y entrevistadores por aportar transparencia, oportunidad y fiabilidad de las evidencias evaluadas. Según Soave y Juaneu (2018) la entrevista es una técnica de indagación altamente valioso y presenta múltiples ventajas sobre otras formas de recoger información respecto de un individuo (p. 2-4), debido a que se puede captar gestos, tonos de voz y otros recursos no verbales que enriquece la entrevista, además que la información recabada es de primera fuente y directa.

Durante la entrevista se debe tener claro el objetivo para desarrollar los campos temáticos, mantener un diálogo dinámico, asertivo y abierto. Las preguntas pueden ser formuladas en tres niveles de análisis: las descriptivas; para averiguar cómo el 
entrevistado realiza sus actividades, las estructurales; para verificar las explicaciones de fenómenos mediáticos y las de contraste; para extraer las diferencias de los términos utilizados por los informantes. Además, Troncoso y Amaya (2016) aseveran que, por medio de la entrevista, el entrevistado queda descubierto con más facilidad dejando conocer y comprender su actuación, considerando aspectos socioculturales de la forma como las personas interpretan la realidad (p. 4).

\section{Instrumento: Guía de preguntas}

Las preguntas sirven para obtener información, revisar procedimientos, fomentar el razonamiento, buscar soluciones, verificar lo aprendido, aplicar nuevas estrategias, reflexionar sobre la actuación y estimular la autoevaluación. Es el planteamiento de preguntas reflexivas pertinentes que sirve como guía para una entrevista, es decir emplear preguntas que requieran pensar; ordenar sus conocimientos o ideas antes de manifestarlos. Estas preguntas ayudan a definir o aclarar conceptos en forma consciente y clara.

Según Troncoso y Amaya (2016), "las preguntas deben ser acompañadas por una breve explicación, lo cual permite guiar en forma adecuada las categorías para aplicar una entrevista. Una vez que se inicia el diálogo, se debe profundizar en las respectivas categorías temáticas" (p. 4). La forma como se estructuran las interrogantes está en relación al tipo de entrevista que se desea realizar: Estructurada; el evaluado recibe un guion de entrevista como el orden de las interrogantes completamente estructuradas. La semiestructurada; delimita la información que resulta de ciertos aspectos relacionados con el objeto de estudio, a fin de obtener datos más profundos en temas definidos, o preguntas abiertas; que permite que quien responda, pueda hilar ideas o explayarse sobre un tema de forma general. Para finalizar se sugiere una pregunta considerando el propósito de la entrevista.

\section{Instrumento: El portafolio virtual}

En la educación superior, el uso del portafolio virtual, también conocido como portafolio electrónico o digital, es cada vez más frecuente sobre todo en un entorno de pandemia donde los institutos de educación superior se enfrentan al reto de acercarse a una preparación por capacidades y educación a distancia. Para Cabero et al. (2018) afirma que, es una herramienta que se basa en una recopilación de sustentos de los métodos de enseñanza y preparación de la misma práctica docente en función de patrones 
determinados (p. 17). Se toman en cuenta la construcción en función de indicadores precisos ya definidos. Resulta ser fundamental para garantizar la calidad en los métodos de enseñanza aprendizaje que se pretende alcanzar.

Debido al estado de emergencia decretado en el Perú, se han fortalecido las practicas con portafolios aplicados en TICs. En este sentido, Ruano y Ballesteros (2017) sostienen que favorece los métodos que se aplican en un portafolio, siendo mejor estructurados, perpetuos y prácticos. Según Yusuf (2017) señala que es una herramienta digital que se pueden desplegar en diversas plataformas tecnológicas, con el fin de agrupar diferentes evidencias del método de enseñanza aprendizaje en diversos medios virtuales, que serán utilizados en su mayoría por los estudiantes (p. 11). Los medios tecnológicos llegan a solucionar problemas de capacidad de almacenamiento de la información, los portafolios virtuales presentan ventajas que van desde ser más accesibles y portables.

Se definen por contribuir a la mejora continua, la toma de decisiones constante, la autoevaluación y posturas críticas sobre las evidencias del desempeño docente. En esta línea Carl y Strydom (2017) afirman que se trata de una herramienta que promueve la reflexión y la conciencia del educador sobre su propio trabajo (p. 24). Por tanto, se trata de una herramienta fundamental, además, de promover la colaboración y discusión profesional entre pares mediante el intercambio de portafolios lo que favorece la comunicación de procedimientos y experiencias.

\section{Figura 3}

Instrumento en la técnica de encuesta

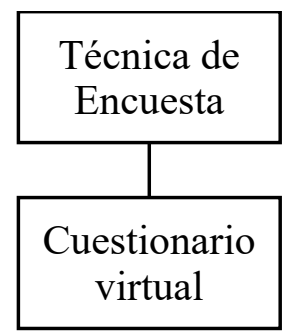

Fuente: Elaboración propia

\section{Técnica: Encuesta}

Dadas las circunstancias actuales donde la práctica docente se desenvuelve en ambientes virtuales, la necesidad de determinar la eficacia del docente aumenta por tanto para que la evaluación del desempeño docente sea justa, integral y con propósitos 
formativos es indispensable aplicar esta técnica, teniendo en cuenta que la finalidad de la evaluación debe ser la mejora de la enseñanza y no solo calificar al maestro.

La encuesta es una técnica que, si está diseñada correctamente y cumple con los criterios psicométricos, va a recopilar la opinión de los receptores de la labor docente. Según Celis et al. (2018), esta técnica presenta ventajas como contestar desde un dispositivo electrónico en casa otorgando mayor veracidad a las respuestas, además se puede aplicar masivamente, agregando preguntas abiertas y cuantificando los resultados con algún software adecuado en forma ágil. Por otro lado, presenta ciertas desventajas; limitación principalmente por el anonimato, ya que no existe control riguroso sobre los evaluadores que respondieron las encuestas, y es poco probable que la totalidad de encuestados responda al mismo tiempo (pp. 4-6).

$\mathrm{Su}$ aplicación se realiza a través de plataformas virtuales y deben estar comprendidas en periodos específicos, de tiempo, en forma mensual o trimestral, así como en tipos de coevaluación de pares, de acuerdo el ámbito en que se desarrollará y a qué docentes estarán dirigidas. En ese sentido, la encuesta de evaluación es un instrumento que permite el análisis, revisión y verificación del proceso del desempeño docente que permitirá garantizar una educación de calidad.

\section{Instrumento: Cuestionario}

Anteriormente se realizaban de manera manual, ahora en tiempos de Covid-19, frecuentemente se hacen en línea en forma virtual, el cuestionario en la evaluación del desempeño docente permite analizar los objetivos de aprendizaje del desarrollo de los cursos asignados. Salinas (2017) señala que, un cuestionario virtual se divide en cinco secciones basados a la experiencia, una dimensión didáctica, tecnológica, de soporte técnico, una valoración general y el de otros comentarios (p. 113).

Estas evaluaciones en línea se realizan para transformar y mejorar las prácticas en la docencia, busca reconocer las necesidades educativas y incentivas la reflexión de los profesores sobre su desenvolvimiento profesional, compartiendo las competencias de valoración del desempeño. Según Gómez y Valdés (2019) aseguran que, el cuestionario es un instrumento privilegiado en la evaluación docente (p. 6). En ese sentido, la evaluación a través del instrumento de un cuestionario está orientada a fortalecer las capacidades del docente. 


\section{Resultados}

El presente artículo es el resultado de un procesamiento de datos teóricos, sobre el cimiento de consultas de distintas fuentes sobre la temática, en la que se resaltan, informes de organismos internacionales, artículos indexados, tesis, libros, revistas, y otros, el objetivo de la evaluación del desempeño docente es dar a conocer la importancia de utilizar instrumentos virtuales para garantizar la calidad profesional de los docentes para la educación superior en tiempos de Covid-19.

\section{Conceptualización del Desempeño Docente}

El desempeño docente, resulta trascendental para enfrentar nuevos retos que implican cambios sociales, culturales y tecnológicos en un entorno de COVID-19. Existen debilidades en la praxis del docente.

En este escenario, la evaluación docente es una preocupación alarmante para el crecimiento del país, las exigencias sobre la aplicación de recursos tecnológicos para la evaluación son indispensables, mencionando a Martínez Rizo (2016), señala que “el fin último de la evaluación del desempeño es mejorar la calidad de la docencia y favorecer el aprendizaje de los estudiantes" (p. 15).

\section{Competencias Pedagógicas}

Las competencias pedagógicas del docente responden a las exigencias de la sociedad actual, logran que el docente se desarrolle con eficacia y eficiencia, reaccionando con rapidez ante situaciones imprevistas.

\section{Competencias Sociales}

Es probable que los alumnos alcancen a desarrollar sentimientos de soledad y abandono, el docente debe poseer habilidades necesarias para orientar el proceso de enseñanza aprendizaje a un entorno amigable, buscar relaciones sanas, impulsando el espacio social.

\section{Competencias Digitales}

La situación sanitaria y social ha puesto en el radar educativo, la digitalización de la enseñanza, la puesta en práctica de conocimientos acerca de las didácticas en entornos digitales.

\section{Técnicas e instrumentos}

Las técnicas e instrumentos son las herramientas de la evaluación del desempeño 
docente, con la finalidad de recoger, analizar e identificar la información adecuada que permita asegurar la correcta ejecución del proceso de enseñanza y aprendizaje de la educación superior en tiempos de Covid-19, según las necesidades que se presenten.

\section{Conclusiones}

En el actual estado de emergencia a consecuencia del Covid-19, las entidades educativas se encuentran en la necesidad de preservar la calidad de la educación; conjunto de condiciones distintas que aseguren, en su interrelación, el correcto funcionamiento de una institución educativa, con la finalidad de lograr la satisfacción de usuarios, excelencia del sistema educativo, eficacia y eficiencia en el proceso, óptimos resultados académicos y un impacto positivo en la sociedad.

La evaluación del desempeño del docente, refiriéndose al proceso continuo y sistemático de recojo de información mediante diversas técnicas e instrumentos con el propósito de emitir un juicio, es un componente indispensable y necesario, que involucra su formación permanente, en distintos contextos, épocas y procesos, convirtiéndose en el actor principal asociado de forma directa a la calidad de la educación.

Las competencias a evaluar en el desempeño docente representan los conocimientos, habilidades, destrezas, actitudes y valores del educador, como también sus capacidades y experiencias en un entorno virtual por motivo de la pandemia mundial. Se clasifica en competencias pedagógicas, sociales y digitales.

La técnica de observación permite una evaluación directa, racional, planificada y sistemática de la práctica docente, en la cual se debe evitar prejuicios, estereotipos, críticas anticipadas que puedan influenciar el resultado de la evaluación a través los instrumentos de la guía de observación, lista de cotejo y la rúbrica.

La técnica de entrevista aportar transparencia, oportunidad y fiabilidad de las evidencias evaluadas. una técnica de indagación de primera fuente, directa, basada en preguntas; estructuradas, semiestructuradas o abiertas, con la finalidad de obtener información, revisar procedimientos, fomentar el razonamiento, buscar soluciones, verificar lo aprendido, aplicar nuevas estrategias, reflexionar sobre la actuación y estimular la autoevaluación. Comprende los instrumentos: guía de preguntas y portafolio virtual.

La técnica de encuesta va a recopilar la opinión de los estudiantes como receptores de la labor docente se desarrolla en la actualidad mediante plataformas virtuales y es 
considerada un complemento necesario para una evaluación del desempeño docente mediante el instrumento del cuestionario.

\section{Referencias}

Achcaray, L., Gámez A. (2018). El liderazgo pedagógico del director y las competencias sociales del personal docente en Instituciones Educativas rurales de la Provincia de Ica [Tesis de Maestría]. Repositorio de la Universidad Nacional de Educación Enrique Guzmán y Valle. http://200.60.81.165/bitstream/handle/UNE/2161/TM\%20CE-

Ge\%203879\%20A1\%20-

$\% 20$ Achcaray $\% 20$ Auris.pdf? sequence $=1 \&$ is Allowed $=\mathrm{y}$

Álvarez, G. (2020). Evaluación del desempeño docente en Instituciones Educativas Públicas. EDUSER, 7(1), 32-40.

Balzola, A. (2020). L'educazione a distanza alla luce e all'ombra della pandemia. Mediascapes journal. https://annalidibotanica.uniroma1.it/index.php/mediascapes/article/view/17126

Burnage, S. (2018). Inspection and observation: A checklist for class teachers. Seced, 2018(23), 6-6. https://doi.org/10.12968/sece.2018.23.6

Cabero J., Llorente M. y Morales, J. (2018). Evaluación del desempeño docente en la formación virtual: ideas para la configuración de un modelo. Revista Iberoamericana de Educación a Distancia RIED. 21 (1), 261-279. https://idus.us.es/bitstream/handle/11441/66943/17206-40396-1$\underline{\mathrm{PB} \% 20 \% 281 \% 29 . p d f ? \text { sequence }=1 \& \text { isAllowed }=\mathrm{y}}$

Carl, A. \& Strydom, S. (2017). e-Portfolio as reflection tool during teaching practice: The interplay between contextual and dispositional variables. South African Journal of Education, 37(1).

Celis, E., Dehesa, E. y Martínez, A (2018). El residente como evaluador del desempeño docente en las especialidades médicas. ScienceDirect. https://doi.org/10.1016/j.edumed.2017.03.021

Cohen, J. y Goldhaber, D. (2016). Building a more complete understanding of teacher evaluation using classroom observations. Educational Researcher, 45(6), 378- 
387. https://journals.sagepub.com/doi/full/10.3102/0013189X16659442

Dorantes, J. y Tobón, S. (2017). Instrumentos de Evaluación: Rúbricas Socioformativas. Praxis Investigativa

ReDIE. https://dialnet.unirioja.es/servlet/articulo? codigo $=6560025$

Durán M., Porlán I. y Prendes M. (2016). Análisis conceptual de modelos de competencia digital del profesorado universitario. RELATEC: Revista Latinoamericana de Tecnología Educativa, 15(1), 97-114. https://dialnet.unirioja.es/servlet/articulo? codigo $=5514576$

Escudero, J., Cutanda, M. y Trillo, J. (2017). Aprendizaje docente y desarrollo profesional del profesorado. Profesorado, Revista de Currículum y Formación del Profesorado, 21(3), 83-102. https://www.redalyc.org/pdf/567/56752489005.pdf

Freire, P. (2017). Pedagogia da autonomia; Saberes necessários à prática pedagógica. Siglo XXI Editores. https://www.researchgate.net/publication/283505639

Geier, M. (2020). The Teacher Behavior Checklist: The Mediation Role of Teacher Behaviors in the Relationship Between the Students' Importance of Teacher Behaviors and Students' Effort. Teaching of Psychology. https://doi.org/10.1177/0098628320979896

Goleman, D. (2018). Inteligencia emocional en la empresa (Imprescindibles). Conecta.

Gómez L. y Valdés M. (2019). The Evaluation of Teacher Performance in Higher Education. Journal of Educational Psychology-Propositos y Representaciones, 7(2), 499-515. http://dx.doi.org/10.20511/pyr2019.v7n2.255

Gonzales, M. (2020). Acompañamiento pedagógico y planificación curricular en docentes de nivel inicial de la red 13 El Agustino. 2020.

Granados, O. (2018). Reforma educativa. FCE - Fondo de Cultura Económica. https://elibro.net/es/lc/bibliotecavirtualidat/titulos/37811

Gunn, A. (2018). Metrics and methodologies for measuring teaching quality in higher education: developing the Teaching Excellence Framework (TEF). Educational Review, 70(2), 129-148. https://doi.org/10.1080/00131911.2017.1410106

Hernández, J. (2016). Rúbricas socioformativas: evaluar para mejorar. Revista Multidiversidad Management.

Hernández, S., Tobón, S. y Guerrero, G. (2016). Hacia una evaluación integral del 
desempeño: las rúbricas socioformativas. Universidad Nacional Autónoma de México (UNAM). https://www.redalyc.org/pdf/461/46148194025.pdf

Instituto Nacional de Tecnologías Educativas y de Formación del profesorado (2017). Competencias digitales docente octubre 2017. Ministerio de Educación, Cultura y Deporte del Gobierno de España. https://aprende.intef.es/sites/default/files/2018-05/2017_1020_MarcoCom $\%$ C3\%BAn-de-Competencia-Digital-Docente.pdf

Kidd, W. \& Murray, J. (2020). The Covid-19 pandemic and its effects on teacher education in England: How teacher educators moved practicum learning online. European Journal of Teacher Education,43(4), 542-558. https://doi.org/10.1080/02619768.2020.1820480

Leiva, M., Montecinos, C. y Aravena, F. (2016). Liderazgo pedagógico en directores nóveles en Chile: Prácticas de observación de clases y retroalimentación a profesores. RELIEVE. Revista Electrónica de Investigación y Evaluación Educativa, 22(2), 1-17. https://www.redalyc.org/pdf/916/91649685008.pdf

Lezcano, L. y Vilanova, G. (2017). Instrumentos de evaluación de aprendizaje en entornos virtuales. Perspectiva de estudiantes y aportes de docentes. Informes Cientificos Técnicos-UNPA,9(1), 1-36. https://doi.org/10.22305/ictunpa.v9i1.235

Lia, M. (2016). Using an observation coaching checklist to provide feedback to teachers. Journal of Catholic Education, 20(1), 311-323. http://dx.doi.org/10.15365/joce.2001152016

Martínez Rizo, F. (2016). La Evaluación de docentes de educación superior. Una revisión de la experiencia internacional. Instituto Nacional para la evaluación de la Educación. https://historico.mejoredu.gob.mx/publicaciones/la-evaluacion-dedocentes-de educacion-basica-una-revision-de-la-experiencia-internacional/

Martínez Ruiz, S. y Lavín, J. (2017) Aproximación al concepto de desempeño docente, una revisión conceptual sobre su delimitación. México. 2-5. http://www.comie.org.mx/congreso/memoriaelectronica/v14/doc/2657.pdf

Martínez G. y Guevara A. (2018). Construcción y validación de un instrumento para evaluar el desempeño docente. RECIE. Revista Electrónica Científica de Investigación Educativa, 4(1), 95-109. 
Organización de las Naciones Unidas para la Educación, la Ciencia y la Cultura (01 de abril de 2020). Aprendiendo en casa: educación a distancia para todos. https://es.unesco.org/news/ aprendiendo-casa-educación-distancia-todos

Palacios J., Ortiz, J., Nuñez, J. y Porras, I. (2019). Competencias sociales en docentes universitarios en la ciudad de Bogotá. Conocimiento global, 4(2), 57-68. http://conocimientoglobal.org/revista/index.php/cglobal/article/view/45

Paz L. (2019). El problema de la instrumentalización de la evaluación del desempeño docente. HELIOS, 2(2). http://200.62.226.189/Helios/article/viewFile/1041/949

Phillips, D. (2018). Many functions of evaluation in education. Education policy analysis archives. http://doi.org/10.14507/epaa.26.3811

Romao, J. (2019). Avaliação na educação: por que a moda? Revista educación, politica y sociedad. https://dialnet.unirioja.es/servlet/articulo? codigo $=6817327$

Ruano, M. y Ballesteros, E. (2017). El portafolio virtual y su relevancia en trabajos colaborativos. Revista Docencia Universitaria, 18(2), 53-68. https://revistas.uis.edu.co/index.php/revistadocencia/article/view/9308/9348

Salinas, M. (2017). Gestión de la evaluación del desempeño docente en aulas virtuales de un proyecto de blended-learning. Ciencia, docencia y tecnología, 28(54), 100-129. https://www.redalyc.org/pdf/145/14551170004.pdf

Saucedo, M. (2018). Implementación de Instrumentos de Evaluación por Competencias, para mejorar la calidad académica en la Carrera de Derecho de la Universidad $\begin{array}{llll}\text { Mayor de San } & \text { Simón. }\end{array}$ http://ddigital.umss.edu.bo:8080/jspui/handle/123456789/10172

Soave, M. y Juaneu, L. (2018). Entrevistas de admisión: Implementación y características teórico técnico. Revista Temas. https://doi.org/10.15332/rt.v0i12.2032

Troncoso, C. y Amaya, A. (2016). Entrevista: guía práctica para la recolección de datos cualitativos en investigación de salud. Rev. Fac. Med. 2017;65: 329-32. https://doi.org/10.15446/revfacmed.v65n2.60235

UNESCO (2020). The Peruvian education system: seeking quality and equity during COVID-19 times. https://historico.mejoredu.gob.mx/publicaciones/laevaluacion-de-docentes-de-educacion-basica-una-revision-de-la-experiencia- 
internacional/

Yusuf, B. (2017). Using an E-Portfolio for Teaching and Teacher Continuous Learning: A Process for Professional Development Enhancement. In Teacher Empowerment Toward Professional Development and Practices. https://doi.org/10.1007/978-981-10-4151-8_20

Giannini, S. (2020). Covid-19 y educación superior: De los efectos inmediatos al día después. Revista Latinoamericana de Educación Comparada, 11(17), 1-57. http://www.iesalc.unesco.org/wp-content/uploads/2020/05/COVID-19-ES130520.pdf

Crawford, J., Butler, K., Rudolph, J., Malkawi, B., Glowattz, M. \& Burton, R. (2020). COVID-19: 20 countries' higher education intra-period digital pedagogy responses. Journal of applied learning \& teaching, 3(1), 1-20. https://doi. org/10.37074/jalt.2020.3.1.7

Cano, M. (2016). Impacto del blended learning en la educación superior. Atenas, 3(31), 55-62. https://www.redalyc.org/pdf/4780/478047207006.pdf

Scull, J., Phillips, M., Sharma, U., \& Garnier, K. (2020). Innovations in teacher education at the time of COVID19: an Australian perspective. Journal of Education for Teaching, 46(4), 497-506. https://doi.org/10.1080/02607476.2020.1802701

García F. (2020). Modelo de referencia para la enseñanza no presencial en universidades presenciales. Campus Virtuales, 9(1), 41-56. www.revistacampusvirtuales.es 


\section{Anexos}

Textos revisados para el artículo:

\begin{tabular}{|c|l|c|c|c|c|c|}
\hline $\mathrm{N}^{\circ}$ & \multicolumn{1}{|c|}{$\begin{array}{c}\text { Base de datos } \\
\text { consultados }\end{array}$} & Libros & Artículos & Tesis & $\begin{array}{c}\text { Página } \\
\text { en un } \\
\text { sitio web }\end{array}$ & Total \\
\hline 1 & COMIE & 0 & 1 & 0 & 0 & 1 \\
\hline 2 & Conocimiento global & 0 & 2 & 0 & 0 & 2 \\
\hline 3 & Dialnet & 0 & 5 & 2 & 0 & 7 \\
\hline 4 & E-libro & 2 & 0 & 0 & 0 & 2 \\
\hline 5 & Fondo editorial INEEN & 1 & 0 & 0 & 0 & 1 \\
\hline 6 & INTEF & 0 & 1 & 0 & 0 & 1 \\
\hline 7 & Libro físico & 1 & 0 & 0 & 0 & 1 \\
\hline 8 & MINEDU & 0 & 0 & 0 & 1 & 1 \\
\hline 9 & Redalyc & 0 & 9 & 0 & 0 & 9 \\
\hline 10 & Rediech & 0 & 2 & 0 & 0 & 2 \\
\hline 11 & Repositorio Universitario & 0 & 3 & 2 & 0 & 5 \\
\hline 12 & Researchgate & 1 & 2 & 0 & 0 & 3 \\
\hline 13 & Revista Eduser & 0 & 1 & 0 & 0 & 1 \\
\hline 14 & Scielo & 0 & 8 & 0 & 0 & 8 \\
\hline 15 & Science Direct & 0 & 3 & 0 & 0 & 3 \\
\hline 16 & Scopus & 0 & 5 & 1 & 0 & 6 \\
\hline 17 & Springer & 0 & 3 & 0 & 0 & 3 \\
\hline 18 & UNESCO & 0 & 0 & 0 & 2 & 2 \\
\hline & $\quad$ Total & 5 & 45 & 5 & 3 & 58 \\
\hline
\end{tabular}

Temporalidad e idioma de los textos revisados para el artículo

\begin{tabular}{|l|l|c|c|c|c|c|c|c|}
\hline \multirow{2}{*}{$\mathrm{N}^{\circ}$} & \multirow{2}{*}{ Textos } & \multicolumn{2}{|c|}{ Temporalidad (antigüedad) } & \multicolumn{5}{c|}{ Idioma } \\
\cline { 2 - 9 } & $\begin{array}{c}0-3 \\
\text { años }\end{array}$ & $\begin{array}{c}4 \mathrm{y} \\
5 \mathrm{annos}\end{array}$ & $\begin{array}{c}6 \mathrm{y} 10 \\
\text { años }\end{array}$ & Español & Inglés & Portugués & Italiano \\
\hline 1 & Libros & 2 & 3 & 0 & 4 & 0 & 1 & 0 \\
\hline 2 & Artículos & 24 & 21 & 0 & 29 & 14 & 1 & 1 \\
\hline 3 & Tesis & 2 & 3 & 0 & 3 & 1 & 0 & 1 \\
\hline 4 & $\begin{array}{l}\text { Página en } \\
\text { un sitio web }\end{array}$ & 3 & 0 & 0 & 3 & 0 & 0 & 0 \\
\hline 4 & Otros... & 0 & 0 & 0 & 0 & 0 & 0 & 0 \\
\hline
\end{tabular}

\section{Textos seleccionados para el artículo:}

\begin{tabular}{|l|l|l|l|l|l|l|}
\hline $\mathrm{N}^{\circ}$ & $\begin{array}{l}\text { Base de datos } \\
\text { consultados }\end{array}$ & Libros & Artículos & Tesis & $\begin{array}{l}\text { Página } \\
\text { en un } \\
\text { sitio web }\end{array}$ & Total \\
\hline 1 & COMIE & 0 & 1 & 0 & 0 & 1 \\
\hline 2 & Conocimiento global & 0 & 1 & 0 & 0 & 1 \\
\hline 3 & Dialnet & 0 & 4 & 1 & 0 & 5 \\
\hline 4 & E-libro & 1 & 0 & 0 & 0 & 1 \\
\hline 5 & Fondo editorial INEE & 1 & 0 & 0 & 0 & 1 \\
\hline 6 & INTEF & 0 & 1 & 0 & 0 & 1 \\
\hline
\end{tabular}




\begin{tabular}{|l|l|l|l|l|l|l|}
\hline 7 & Libro físico & 1 & 0 & 0 & 0 & 1 \\
\hline 8 & MINEDU & 0 & 0 & 0 & 1 & 1 \\
\hline 9 & Redalyc & 0 & 8 & 0 & 0 & 8 \\
\hline 10 & Rediech & 0 & 1 & 0 & 0 & 1 \\
\hline 11 & Repositorio Universitario & 0 & 2 & 1 & 0 & 3 \\
\hline 12 & Researchgate & 1 & 1 & 0 & 0 & 2 \\
\hline 13 & Revista Eduser & 0 & 1 & 0 & 0 & 1 \\
\hline 14 & Scielo & 0 & 6 & 0 & 0 & 6 \\
\hline 15 & Science Direct & 0 & 2 & 0 & 0 & 2 \\
\hline 16 & Scopus & 0 & 3 & 0 & 0 & 3 \\
\hline 17 & Springer & 0 & 1 & 0 & 0 & 1 \\
\hline 18 & UNESCO & 0 & 0 & 0 & 2 & 2 \\
\hline \multicolumn{2}{|l|}{ Total } & 4 & 32 & 2 & 3 & 41 \\
\hline
\end{tabular}

Temporalidad e idioma de los textos seleccionados para el artículo

\begin{tabular}{|l|l|c|c|c|c|c|c|c|}
\hline \multirow{2}{*}{$\mathrm{N}^{\circ}$} & \multirow{2}{*}{ Textos } & \multicolumn{3}{|c|}{$\begin{array}{c}\text { Temporalidad } \\
\text { antigüedad) }\end{array}$} & \multicolumn{4}{c|}{ Idioma } \\
\cline { 2 - 9 } & $\begin{array}{c}0-3 \\
\text { años }\end{array}$ & $\begin{array}{c}4 \text { y } \\
5 \text { años }\end{array}$ & $\begin{array}{c}6 \text { y } 10 \\
\text { años }\end{array}$ & Español & Inglés & Portugués & Italiano \\
\hline 1 & Libros & 2 & 2 & 0 & 3 & 0 & 1 & 0 \\
\hline 2 & Artículos & 16 & 16 & 0 & 20 & 10 & 1 & 1 \\
\hline 3 & Tesis & 2 & 0 & 0 & 2 & 0 & 0 & 0 \\
\hline 4 & $\begin{array}{l}\text { Página en } \\
\text { un sitio } \\
\text { web }\end{array}$ & 3 & 0 & 0 & 3 & 0 & 0 & 0 \\
\hline 4 & Otros & 0 & 0 & 0 & 0 & 0 & 0 & 0 \\
\hline
\end{tabular}

